\title{
APOBEC3B expression and its prognostic potential in breast cancer
}

\author{
YAN MAO $^{1}$, MENG LV $^{1}$, YUZI ZHANG ${ }^{2}$, GANG NIE $^{1}$, JIAN CUI $^{1}$, YONGMEI WANG $^{1}$, \\ YUANYUAN WANG ${ }^{1}$, WEIHONG CAO ${ }^{1}$, XIAOYI LIU ${ }^{1}$, XINGANG WANG $^{1}$ and HAIBO WANG ${ }^{1}$ \\ ${ }^{1}$ Breast Disease Center, The Affiliated Hospital of Qingdao University, Qingdao, Shandong 266003; \\ ${ }^{2} 3$ D Medicines Inc., Shanghai 200025, P.R. China
}

Received July 13, 2019; Accepted January 3, 2020

DOI: $10.3892 / 01.2020 .11433$

\begin{abstract}
Apolipoprotein B mRNA editing enzyme catalytic subunit 3B (APOBEC3B) mRNA expression is associated with the poor prognosis of estrogen receptor positive $(\mathrm{ER}+)$ breast cancer. However, the clinical relevance of APOBEC 3B protein expression in patients with breast cancer remains unclear. The present study evaluated the association of APOBEC3B protein expression with clinicopathological features, as well as survival outcomes of patients with breast cancer. Furthermore, the association between APOBEC3B protein expression and tumor infiltrating lymphocytes (TILs) was investigated. APOBEC3B protein expression in 120 patients with breast cancer was evaluated via immunohistochemistry, using a constructed tumor microarray, and TILs were analyzed by hematoxylin and eosin staining. The relevance of APOBEC3B mRNA expression in breast cancer was assessed using a Kaplan-Meier Plotter online tool, as well as the Tumor Immune Estimation Response and The Cancer Genome Atlas databases. The present study assessed APOBEC3B expression in 116 patients with breast cancer and demonstrated that protein expression was significantly associated with ER and progesterone receptor expression, as well as different subtypes of breast cancer. Notably, APOEBC3B protein expression was significantly associated with TILs. Overall, high expression levels of APOBEC3B protein and high levels of TILs were indicative of longer disease-free survival rate. High APOBEC3B mRNA expression was associated with poor relapse-free survival rate, overall survival rate and distant metastasis-free survival rate in patients with breast cancer, particularly for the Luminal A subtype. APOBEC3B mRNA expression was also indicated to be associated with the immune
\end{abstract}

Correspondence to: Dr Yan Mao or Dr Haibo Wang, Breast Disease Center, The Affiliated Hospital of Qingdao University, 16 Jiangsu Road, Qingdao, Shandong 266003, P.R. China

E-mail: 1012052081@qq.com

E-mail: hbwang66@126.com

Key words: apolipoprotein B mRNA editing enzyme catalytic subunit 3B, breast cancer, immune, survival status of patients with breast cancer. Overall, the results of the present study demonstrated that APOBEC3B mRNA and protein expression levels presented different prognostic values in the survival of patients with breast cancer. However, both APOBEC3B mRNA and protein expression levels were associated with TILs in breast cancer. Therefore, APOBEC3B may be a prognostic biomarker for breast cancer.

\section{Introduction}

Breast cancer, one of the most common malignancies among women (11.6\% of total cancer cases in 2018), is a confounding heterogeneous disease (1-3). Genetic mutations have been demonstrated to serve a crucial role in the development and progression of several types of cancer, including breast cancer (4-7). Previous genome sequencing studies have indicated that clustered mutations contribute to breast cancer progression $(6,8)$, which may explain the poor response to single target therapy. Consequently, clustered mutations may pose as biomarkers for the prognosis of breast cancer. Overall, it is critical to develop novel prognostic biomarkers, in order to improve precision therapy of breast cancer.

The Apolipoprotein B mRNA editing enzyme, catalytic polypeptide-like (APOBEC) protein family is a large family of evolutionarily conserved cytidine deaminases (9). APOBECs serve an important role in the innate immune system, protecting against viral pathogens, such as restriction of HIV-1 viral reverse transcription (10-14). Notably, apolipoprotein B mRNA editing enzyme catalytic subunit 3B (APOBEC3B), a member of the APOBEC family, has been demonstrated to induce somatic mutations in several types of malignancies, including breast cancer (15-21). This suggests that APOBEC3B may be a key factor for clustered mutations in cancer. A number of studies have demonstrated that APOBEC3B mRNA expression levels are upregulated in breast cancer, which is associated with the metastasis, endocrine therapy resistance and poor prognosis of patients with estrogen receptor positive (ER+) breast cancer (22-25); however, to the best of our knowledge, the clinical relevance of the APOBEC3B protein in breast cancer has not yet been determined. Therefore, the present study aimed to investigate the role of APOBEC3B protein expression in breast cancer. The results were inconclusive with regards to the significance of APOBEC $3 \mathrm{~B}$ mRNA 
expression levels in breast cancer, particularly for patients who received endocrine therapy. Furthermore, related analyses were performed especially in patients who received a different treatment with publicly available data. As APOBEC3B is well-known for its involvement in innate immunity and is also associated with tumor infiltrating lymphocytes (TILs) $(14,26)$, associations between APOBEC3B and immune biomarkers of breast cancer were also assessed.

\section{Materials and methods}

Patients and tissue samples. Clinicopathological data were collected from 120 female patients who received breast cancer surgery at The Affiliated Hospital of Qingdao University (Qingdao, China) between February 2008 and November 2010. The median age of the patients was 56 with an age range of 23-85 years. The patients were followed-up from the date of diagnosis until May 2018 or mortality. Follow-up began 6 months after surgery and was performed by outpatient examination and/or by telephone every 3 months. The present study was approved by the Ethics Committee of The Affiliated Hospital of Qingdao University and all patients provided written informed consent prior to enrolment in the study. TNM stage was classified according to the American Joint Committee on Cancer guidelines, 7th Edition (27).

Formalin-fixed and paraffin-embedded blocks of the 120 breast cancer samples were retrospectively collected and from each block, a representative 1-mm-diameter core of tissue was selected, re-arrayed and re-embedded in a recipient block to prepare the tissue microarrays (TMAs). In order to ensure that cancer tissue was collected, all slides were reviewed using hematoxylin and eosin (H\&E) staining. Hematoxylin staining was performed for $4 \mathrm{~min}$ and eosin staining for $1 \mathrm{~min}$ at room temperature. The most effective coring region was selected for TMA construction. Additionally, the H\&E sections of the recipient blocks were observed following TMA construction, to confirm that the selected region was contained within the cores. The 120 breast cancer specimens were examined via immunohistochemistry for APOBEC3B protein, as previously described (28). Scoring was based on the percentage of stained tumor cells, as follows: $0-10 \%$, negative (-); 11-25\%, slightly positive (+); 26-50\%, moderately positive $(++)$; and $51-100 \%$, strongly positive $(+++)(29)$. Cores were categorized into low expression (- and +$)$ and high expression $(++$ and +++$)$ for further analyses. All scoring was completed independently and blind by two pathologists (Pathology Group of Breast Disease Center, the Affiliated Hospital of Qingdao University) with agreement between the two $>90 \%$ of the time. Disagreements were resolved by consensus. TILs were assessed using H\&E stained sections and the International TILs Working Group 2014 scoring standard was implemented (30). Furthermore, TILs were assessed independently and blind by the same investigators and the mean values were used for analysis. The prognostic value of APOBEC3B mRNA for breast cancer was determined using the Kaplan-Meier Plotter online tool (http://kmplot.com). The cut-off value for APOBEC3B mRNA expression levels was the median value. The patients with similar prevalence to the SEER program in the Kaplan-Meier Plotter online service were defined as 'SEER similar'.
Reagents and antibodies. Monoclonal primary antibodies against APOBEC3B (1:100; cat. no ab191695; Abcam) were used for IHC staining. Anti-rabbit secondary antibodies were purchased from Abcam (cat. no ab6112; Abcam) and a dilution of 1:500 was used for staining.

Immune cell infiltration analysis. RNA sequencing data and clinical data of 1,102 patients with breast cancer were downloaded from The Cancer Genome Atlas (TCGA) database (https://portal.gdc.cancer.gov). Data on the abundance of CD8 cells, CD4 T cells, CD8 T cells, neutrophils, macrophages and dendritic cells per sample ID were obtained from Li et al (31), which was accessible through the Tumor Immune Estimation Response (TIMER) database (http://cistrome.org/TIMER).

Statistical analysis. Statistical analyses were performed using SPSS software (v.22.0; IBM Corp.). The $\chi^{2}$ test was used to assess the association between APOBEC3B protein expression levels and clinicopathological data. The Kaplan-Meier method and the Cox-regression model were used for survival analysis. Differences between two survival curves was assessed with log-rank test. All analyses were two-sided. $\mathrm{P}<0.05$ was considered to indicate a statistically significant difference. Disease-free survival (DFS) time was defined as the interval (in months) between the date of breast surgery to first recurrence (loco-regional recurrence and/or distant metastasis). Relapse-free survival (RFS) time was defined as the interval (in months) between the date of breast surgery to first loco-regional recurrence. Distant metastasis free survival (DMFS) time was defined as the interval (in months) between the date of breast surgery to first distant metastasis. Overall survival (OS) time was defined as the interval (in months) between the date of breast surgery and breast cancer-associated mortality. The Kruskal-Wallis test was employed to compare the abundance of immune cell infiltrates among the groups. The Dunn's test was used as the post-hoc test following Kruskal-Wallis. The difference between two groups was assessed with Mann-Whitney U test.

\section{Results}

Clinicopathological characteristics of patients with breast cancer. In total, 120 patients who received breast cancer surgery at The Affiliated Hospital of Qingdao University were evaluated in the present study. A total of four specimen were lost during sectioning, thus 116 cases were analyzed. The clinicopathological characteristics of the patients are presented in Table I. The median diagnostic age was 56 years (range, 23-85 years), and the majority of cases were ductal carcinoma $(n=87 ; 75.0 \%)$. The majority of patients presented with histological grade $1(\mathrm{n}=52 ; 44.8 \%)$, followed by grade 2 $(\mathrm{n}=50 ; 43.1 \%)$ and only two patients had T3 (1.7\%; Table I). Regarding pathological characteristics, the majority of patients were classified in stage I $(n=48 ; 41.4 \%)$ and stage II $(n=56$; $48.3 \%)$, and were both $\mathrm{ER}+(\mathrm{n}=86 ; 74.1 \%)$ and progesterone receptor $(\mathrm{PR})+(\mathrm{n}=73 ; 62.9 \%)$. Regarding molecular subtypes, the majority of cases were luminal-like(Luminal A, $\mathrm{n}=50$, $43.1 \%$; Luminal $\mathrm{B}, \mathrm{n}=37,31.9 \%$ ), whereas nine patients were human epidermal growth factor receptor-2 (HER2) positive $(7.8 \%)$. After a median follow-up of 97 months (range, 
Table I. Clinicopathological characteristics of patients with breast cancer $(n=116)$.

\begin{tabular}{lll}
\hline Characteristics & Patients, $n$ & Percentage \\
\hline
\end{tabular}

Age at diagnosis, years

$56(23-85)$

(median range)

Age, years

$$
\leq 65
$$

$>65$

79

37

$97(8-123)$

15

Number of mortalities

Disease-free survival, months

Median, range

Number of recurrences

91 (4-123)

22

Histological subtype

IDC
ILC
Others

Menopausal status

Premenopausal

Postmenopausal

Histological grade, \%

G1

G3

Negative

HER2 status

Positive

Negative

Ki67,\%

$>14$

$\leq 14$

T category

T1

T2

T3

LN status

Negative

Positive

Pathological stage

$$
\text { I }
$$

II

III

Molecular subtype

Luminal A

Luminal B
19.0

68.1

31.9

75.0

10.3

14.7

40.5

59.5

44.8

43.1

12.1

74.1

25.9

62.9

37.1

7.8

92.2

58.6

41.4

50.0

48.3

69.8

30.2

41.4

48.3

10.3

31.9
Table I. Continued.

\begin{tabular}{lcc}
\hline Characteristics & Patients, $\mathrm{n}$ & Percentage \\
\hline HER2 amplification & 3 & 2.6 \\
Triple negative & 26 & 22.4 \\
APOBEC3B protein & & \\
expression & & \\
High & 66 & 56.9 \\
Low & 50 & 43.1 \\
\hline
\end{tabular}

IDC, invasive ductal carcinoma; ILC, invasive lobular carcinoma; ER, estrogen receptor; PR, progesterone receptor; LN, lymph node; APOBEC3B, apolipoprotein B mRNA editing enzyme catalytic subunit 3B.

8.0-123.0 months), 15 patients had died and seven patients had relapsed by the end of the present study (data not shown).

APOBEC3B protein expression by TMA and association with clinicopathological characteristics. APOBEC3B protein staining was performed in accordance with a previous study on ovarian cancer (26) and was localized to both nuclear and cytoplasmic compartments (Fig. 1). Of the 116 patients, 66 demonstrated high levels of APOBEC3B protein expression $(56.9 \%)$. The associations between APOBEC3B protein expression levels and clinicopathological characteristics are presented in Table II. APOBEC3B protein expression was significantly associated with ER and PR expression (both $\mathrm{P}<0.05)$, and with different subtypes of breast cancer $(\mathrm{P}=0.045)$. However, no significant associations were identified between APOBEC3B protein expression and other clinicopathological characteristics such as age, histological grade, tumor size and TNM stage. Notably, no significant associations were identified between APOBEC3B protein expression and DFS time $(\mathrm{P}=0.975)$ or $\mathrm{OS}$ time $(\mathrm{P}=0.400)$ in patients with breast cancer (Fig. 2A and B, respectively).

$A P O B E C 3 B$ protein expression and TILs. The association between APOBEC3B protein expression and TILs was analyzed in the 116 patients with breast cancer, in order to determine the role of APOBEC3B in the immune system. The results demonstrated that high APOBEC3B protein expression was associated with TILs $(\mathrm{P}=0.016)$, particularly TILs within the stroma ( $\mathrm{P}=0.018$; Table II and Fig. S1). Subsequently, patients were divided into four groups: i) High APOBEC3B protein expression and high levels of TILs; ii) high APOBEC $3 B$ protein expression and low levels of TILs; iii) low APOBEC3B protein expression and high levels of TILs; and iv) low APOBEC3B protein expression and low levels of TILs, in order to analyze the effects of APOBEC3B protein expression and TILs on survival. The results demonstrated that patients with high APOBEC3B protein expression and high levels of TILs had improved DFS time ( $\mathrm{P}=0.049)$, with a trend for improved OS time $(\mathrm{P}=0.235)$, compared with patients with high APOBEC3B protein expression and low levels of TILs (Fig. 2C and D, respectively). Multivariate analysis demonstrated that patients in the subgroup with 

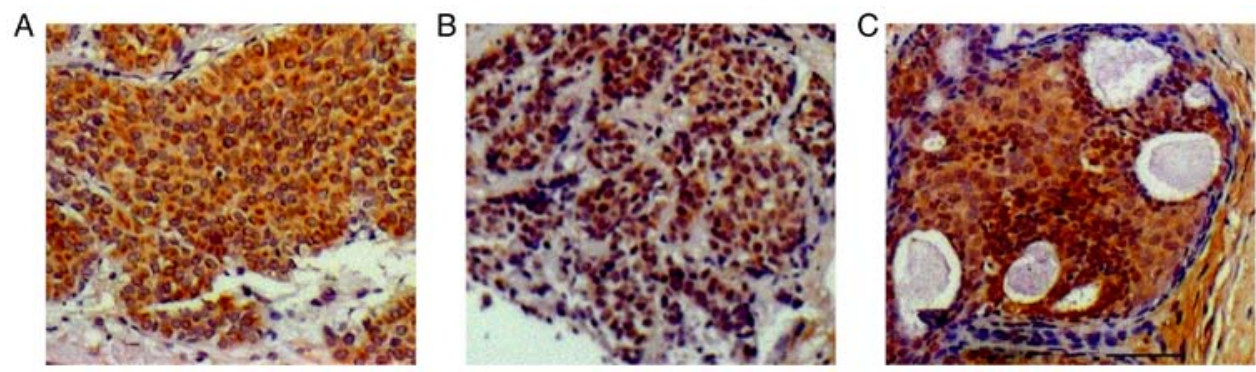

Figure 1. Representative immunohistochemical images of APOBEC3B expression in breast cancer tissue microarrays. APOBEC3B protein expression localized in the (A) cytoplasmic compartment, (B) nuclear compartment and (C) both. Scale bar, $200 \mu \mathrm{m}$. APOBEC3B, apolipoprotein B mRNA editing enzyme catalytic subunit 3B.
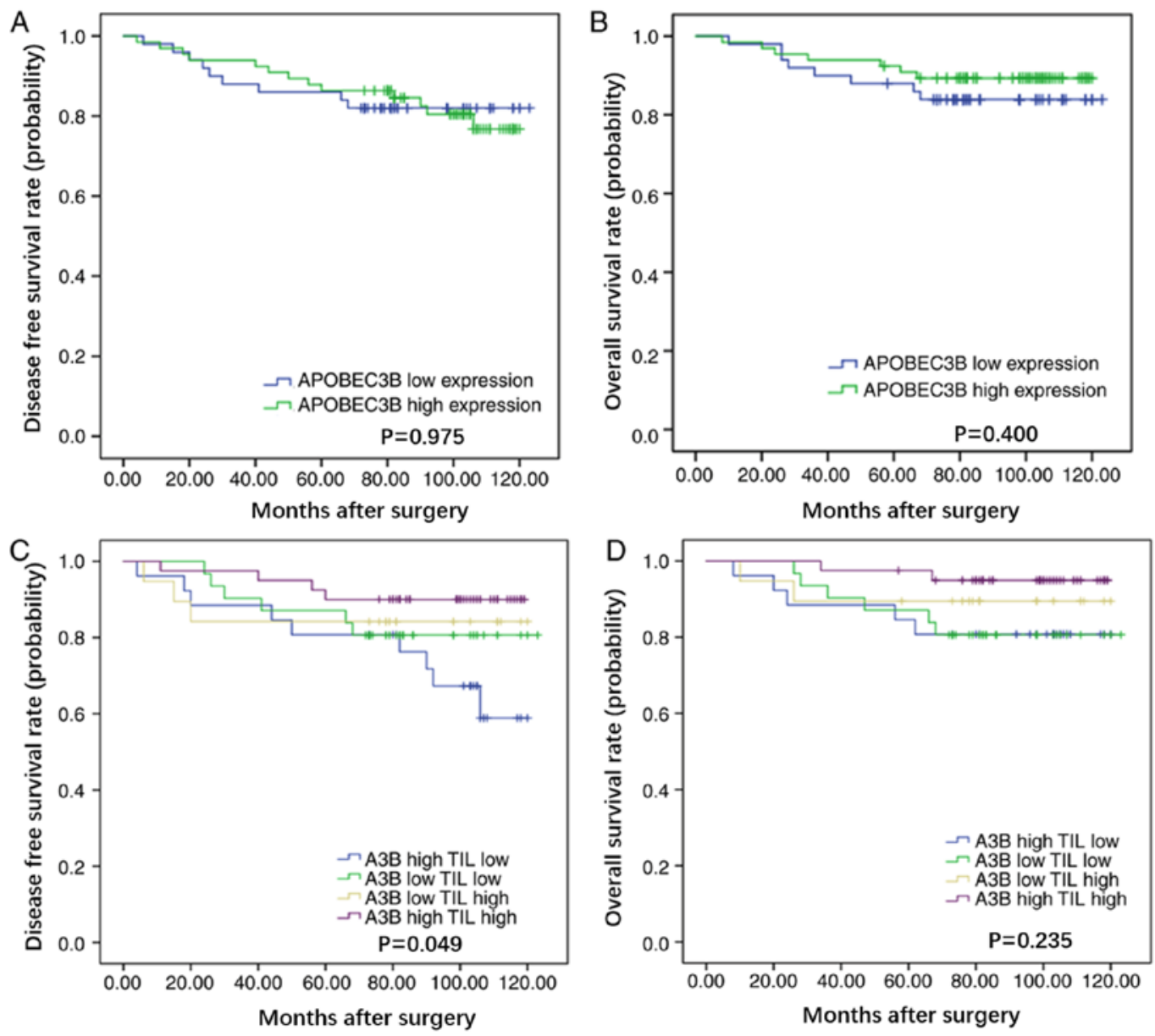

Figure 2. Kaplan-Meier survival curves depicting DFS and OS for the 116 patients breast cancer. (A) DFS curves for patients with different levels of APOBEC3B protein expression. (B) OS curves for patients with different levels of APOBEC3B expression. (C) DFS curves for patients with different levels of combined APOBEC3B protein expression TILs. (D) OS curves for patients with different levels of combined APOBEC3B protein expression and TILS. APOBEC3B, apolipoprotein B mRNA editing enzyme catalytic subunit 3B; TILS, tumor-infiltrating lymphocytes; OS, overall survival; DFS, disease free survival.

high APOBEC3B protein expression and high levels of TILs exhibited improved DFS time compared with patients with high APOBEC3B protein expression and low levels of TILs [hazard ratio $(\mathrm{HR})=0.65 ; 95 \% \mathrm{CI}=0.45-0.95 ; \mathrm{P}=0.024$; Table III].

APOBEC $3 B$ mRNA expression in breast cancer. A total of 3,951 breast cancer cases were assessed using the data in the Kaplan-Meier Plotter online tool. With the median value set as the cut-off value, the results demonstrated that patients with high APOBEC3B mRNA expression levels had poor 10-year relapse-free survival (RFS) rate $(n=3,951 ; \mathrm{HR}=1.64$; 95\% $\mathrm{CI}=1.47-1.83 ; \mathrm{P}<0.00001)$, OS rate $(\mathrm{n}=1,402 ; \mathrm{HR}=1.93$; 95\% $\mathrm{CI}=1.54-2.41 ; \mathrm{P}=0.00084)$ and distant metastasis-free survival (DMFS) rate $(\mathrm{n}=1,746, \mathrm{HR}=1.69 ; 95 \% \mathrm{CI}=1.38-2.07$; $\mathrm{P}=0.00237$; Fig. 3 ). Analysis was also performed in the patients who had a similar prevalence to the SEER (Surveillance, Epidemiology, and End Results) database and similar trends 
Table II. Associations between APOBEC3B protein expression and clinicopathological characteristics of patients with breast cancer.

\begin{tabular}{|c|c|c|c|c|c|c|c|c|c|}
\hline & & & & & & & & & \\
\hline breast cancer. & & & & & & $\begin{array}{l}\text { APOI } \\
\text { expre }\end{array}$ & $\begin{array}{l}\text { C3B } \\
\text { on, n }\end{array}$ & & \\
\hline & $\begin{array}{l}\text { APOI } \\
\text { expre }\end{array}$ & $\begin{array}{l}\text { C3B } \\
\text { ion, } n\end{array}$ & & & Characteristics & High & Low & $\chi^{2}$ & P-value \\
\hline Characteristics & High & Low & $\chi^{2}$ & P-value & sTILs & & & 5.564 & 0.018 \\
\hline Age, years & & & 0.614 & 0.433 & $\begin{array}{l}\text { High } \\
\text { Low }\end{array}$ & $\begin{array}{l}37 \\
29\end{array}$ & $\begin{array}{l}17 \\
33\end{array}$ & & \\
\hline$\leq 65$ & 43 & 36 & & & TILs & & & 5.817 & 0.016 \\
\hline$>65$ & 23 & 14 & & & High & 40 & 19 & & \\
\hline $\begin{array}{l}\text { Histological subtype } \\
\text { IDC }\end{array}$ & 53 & 34 & 2.520 & 0.284 & Low & 26 & 31 & & \\
\hline
\end{tabular}

Others

Menopause status

Premenopausal

Postmenopausal

Histological grade

G1

G2

G3

ER status

Positive

Negative

PR status

Positive

Negative

HER2 status

Positive

Negative

Ki67,\%

$>14$

$\leq 14$

T category

T1

$\mathrm{T} 2$

T3

LN status

Negative

Positive

6

7

30

36

6

10

$$
1.549
$$

0.213

17

33

2.769

0.250

34

25

7

18

7

4.711

0.030

$54 \quad 32$

12

18

$10.799 \quad 0.001$

$50 \quad 23$

$16 \quad 27$

Pathological stage

$$
\text { I }
$$

II

III

Molecular subtype

Luminal A

Luminal B

HER2 amplification

Triple negative

iTILs

High

Low
Table II. Continued.

APOBEC3B, apolipoprotein B mRNA editing enzyme catalytic subunit 3B; IDC, invasive ductal carcinoma; ILC, invasive lobular carcinoma; ER, estrogen receptor; PR, progesterone receptor; HER2, human epidermal growth factor receptor-2; LN, lymph node; iTILs, intratumoral infiltrating lymphocytes; sTILs, tumor infiltrating lymphocytes in stroma; TILs, tumor infiltrating lymphocytes.

were observed for 10 -year RFS rate $(n=493 ; \mathrm{HR}=1.47,95 \%$ $\mathrm{CI}=1.04-2.07 ; \mathrm{P}=0.028)$, OS rate $(\mathrm{n}=301 ; \mathrm{HR}=1.97 ; 95 \%$ $\mathrm{CI}=1.22-3.19 ; \mathrm{P}=0.0047)$ and $\mathrm{DMFS}$ rate $(\mathrm{n}=375 ; \mathrm{HR}=1.54$; 95\% CI=1.02-2.31; $\mathrm{P}=0.036$; Fig. S2). Overall, these results suggest that high APOBEC3B mRNA expression levels are associated with poor prognosis of breast cancer.

Previous studies have reported that high APOBEC3B mRNA expression levels indicate a worse survival in ER+ patients (24-26). The present study analyzed different subtypes of breast cancer and the results demonstrated that the prognostic value of APOBEC3B mRNA expression was significantly associated with the Luminal A subtype in 10-year-RFS rate $(\mathrm{n}=1,933 ; \mathrm{HR}=1.68 ; 95 \% \mathrm{CI}=1.41-2.01 ; \mathrm{P}=0.00084$; Fig. S3A), 10 -year-OS rate $(\mathrm{n}=611 ; \mathrm{HR}=2.3 ; 95 \% \mathrm{CI}=1.55-3.41 ; \mathrm{P}=0.0135$; Fig. 4A) and 10-year-DMFS rate $(\mathrm{n}=965 ; \mathrm{HR}=1.67 ; 95 \%$ $\mathrm{CI}=1.22-2.27$; $\mathrm{P}=0.0011$; Fig. S4A). Conversely, APOBEC3B mRNA expression was only significantly associated with the Luminal B subtype in 10-year-RFS rate $(n=1,149 ; \mathrm{HR}=1.32$; 95\% CI=1.08-1.60; P=0.0056; Fig. S3B).

Similar analyses were performed for patients who did or did not receive systemic therapy. The results for patients that did not receive treatment were as follows: 10-year-RFS rate $(\mathrm{n}=1,010 ; \mathrm{HR}=1.58 ; 95 \% \mathrm{CI}=1.26-1.98 ; \mathrm{P}<0.05)$ and 10 -year-OS rate $(\mathrm{n}=382 ; \mathrm{HR}=1.98 ; 95 \% \mathrm{CI}=1.20-3.28$; $\mathrm{P}=0.0068$; Fig. S5). The results for patients that received endocrine therapy were as follows: 10 -year-RFS rate $(n=929$; $\mathrm{HR}=1.35 ; 95 \% \mathrm{CI}=1.04-1.77 ; \mathrm{P}=0.026)$ and 10 -year-OS rate $(\mathrm{n}=133 ; \mathrm{HR}=2.95 ; 95 \% \mathrm{CI}=1.36-6.38 ; \mathrm{P}=0.0039)$, and those that received tamoxifen: 10 -year-RFS rate $(n=740 ; H R=1.37$; 95\% $\mathrm{CI}=1.00-1.87 ; \mathrm{P}=0.0047)$ and 10 -year-OS rate $(\mathrm{n}=130$; $\mathrm{HR}=2.61 ; 95 \% \mathrm{CI}=1.19-5.74 ; \mathrm{P}=0.013$; Fig. S6). Overall, patients with high APOBEC3B mRNA expression levels were revealed to have worse 10 -year-RFS and OS rates. APOBEC3B mRNA expression has no significant effect on 10-year-RFS rate $(\mathrm{n}=798 ; \mathrm{HR}=1.28 ; 95 \% \mathrm{CI}=0.97-1.67 ; \mathrm{P}=0.075)$ and 10 -year-OS rate $(\mathrm{n}=300 ; \mathrm{HR}=0.99 ; 95 \% \mathrm{CI}=0.61-1.62 ; \mathrm{P}=0.98$; Fig. S6) for patients with chemotherapy. 


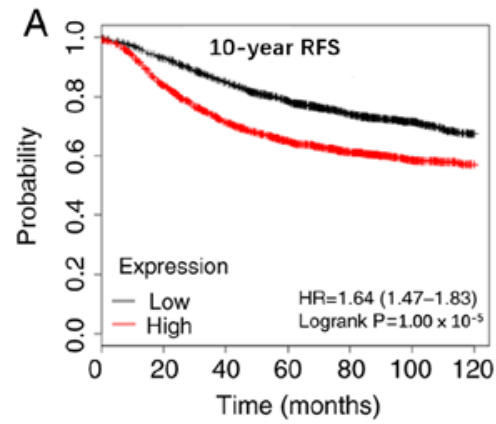

Number at risk

Low 1980180515571277953627401

High 197116041240958708448284

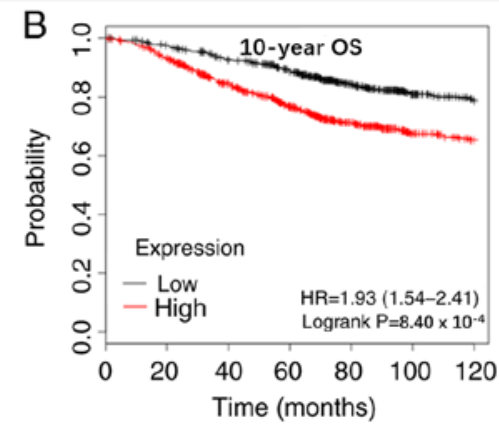

Number at risk

Low $\begin{array}{lllllll}705 & 676 & 623 & 558 & 418 & 293 & 238\end{array}$

High $\begin{array}{lllllll}6975 & 524 & 422 & 298 & 184 & 136\end{array}$

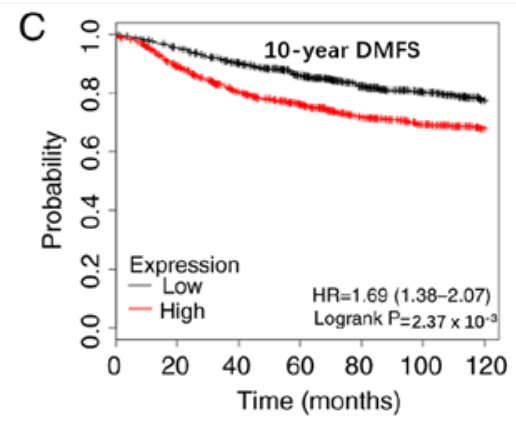

Number at risk

Low $873 \quad 814 \quad 731 \quad 607 \quad 444 \quad 317 \quad 219$

High $873 \quad 744 \quad 607 \quad 496361 \quad 275180$

Figure 3. Kaplan-Meier survival curves for 10-year (A) RFS, (B) OS and (C) DMFS rates, stratified by APOBEC3B mRNA expression in patients with breast cancer. RFS, relapse-free survival; OS, overall survival; DMFS, distant metastasis-free survival; y, years; APOBEC3B, apolipoprotein B mRNA editing enzyme catalytic subunit 3B.
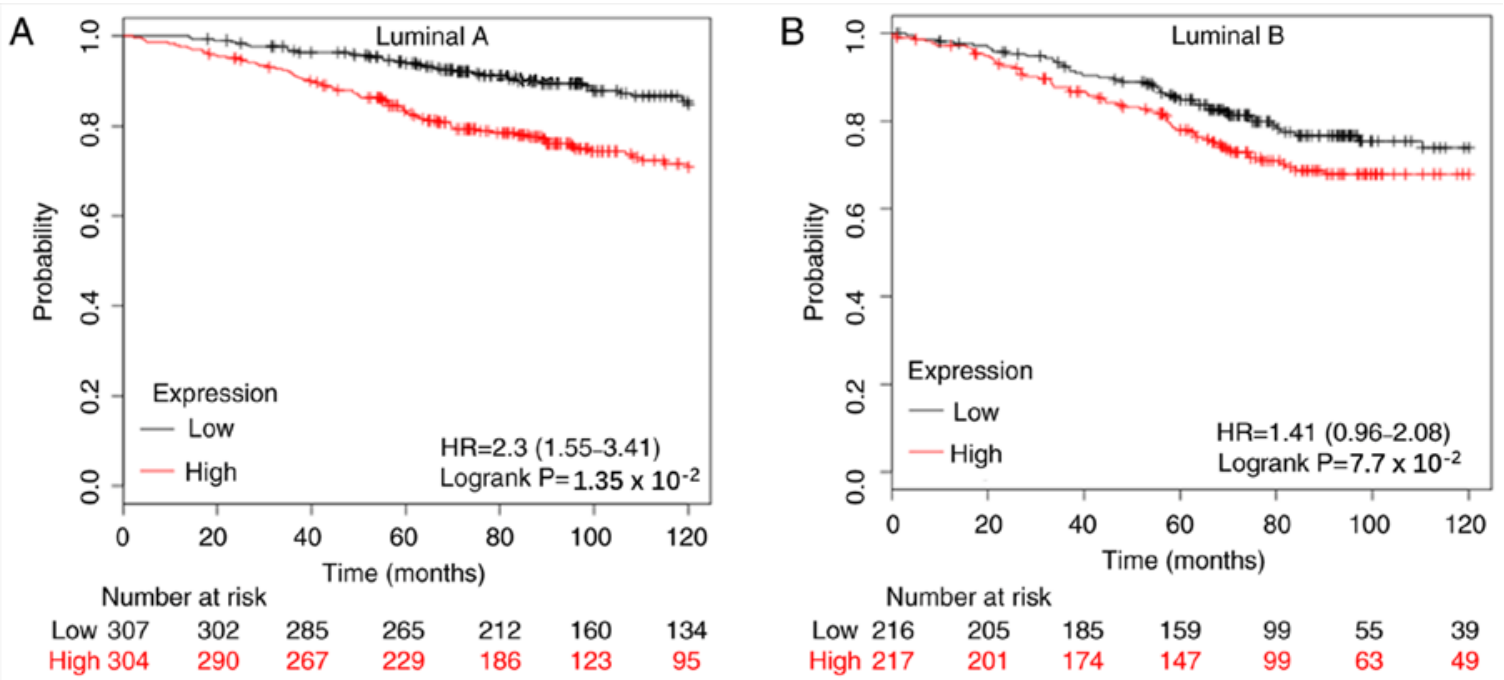

$\begin{array}{lllllll}\text { Low } 216 & 205 & 185 & 159 & 99 & 55 & 39 \\ \text { High } 217 & 201 & 174 & 147 & 99 & 63 & 49\end{array}$
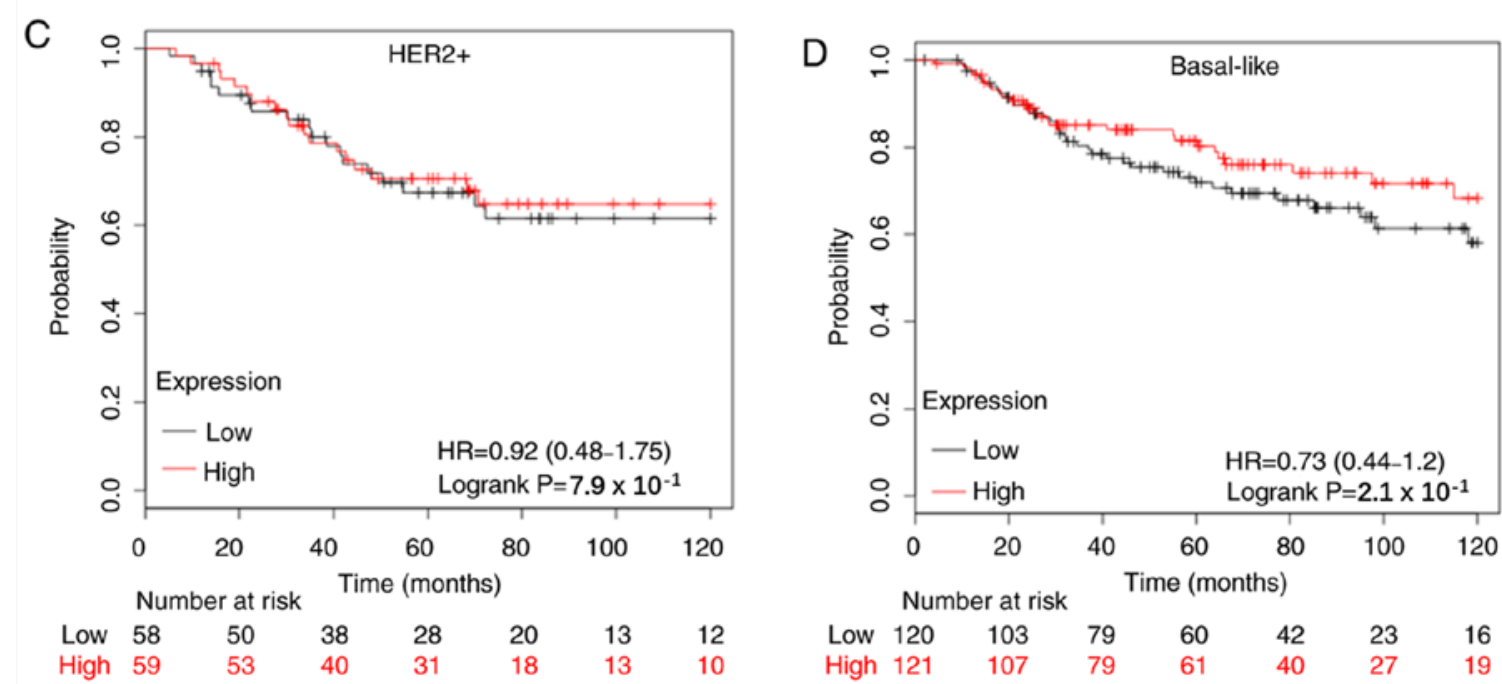

Figure 4. Kaplan-Meier survival curves for 10-year overall survival rate stratified by APOBEC3B mRNA expression for patients with (A) Luminal A, (B) Luminal B, (C) HER2+ and (D) triple negative breast cancer. APOBEC3B, apolipoprotein B mRNA editing enzyme catalytic subunit 3B; HER2+, human epidermal growth factor receptor-2 positive.

APOBEC $3 B$ mRNA and the immune system. The median cut-off value was used in the TCGA database to determine the association between APOBEC3B mRNA expression levels and immune-related genes. The results demonstrated that APOBEC3B mRNA expression levels were positively associated with $\mathrm{B}$-cells $(\mathrm{r}=0.197 ; \mathrm{P}<0.05)$ and dendritic cells $(r=0.155 ; \mathrm{P}<0.05)$; however, a negative trend association was demonstrated with macrophages $(r=-0.115 ; \mathrm{P}>0.05$; 
Table III. Univariate and multivariate analyses of characteristics associated with DFS.

DFS time

\begin{tabular}{|c|c|c|}
\hline Characteristics & HR $(95 \% \mathrm{CI})$ & P-value \\
\hline \multicolumn{3}{|l|}{ Univariate } \\
\hline Age, years ( $\leq 65$ vs. $>65)$ & $1.95(0.84-4.51)$ & 0.120 \\
\hline Histological subtype (IDC vs. Others) & $0.84(0.44-1.58)$ & 0.582 \\
\hline Grade (1 vs. $2 / 3)$ & $0.29(0.11-0.73)$ & 0.009 \\
\hline Tumor size, cm (>5 vs. $\leq 5)$ & $4.04(1.49-10.98)$ & 0.006 \\
\hline Lymph node (+ vs. -) & $3.00(1.29-7.01)$ & 0.011 \\
\hline TNM stage (II/III vs. I) & $2.09(1.13-3.87)$ & 0.019 \\
\hline ER (+ vs. - ) & $0.68(0.28-1.67)$ & 0.402 \\
\hline PR (+ vs. -) & $0.62(0.26-1.43)$ & 0.260 \\
\hline HER2 (+ vs. -) & $3.65(1.22-10.92)$ & 0.020 \\
\hline Ki67, \% ( $\leq 14$ vs. $>14)$ & $0.78(0.33-1.87)$ & 0.581 \\
\hline \multicolumn{2}{|l|}{ Molecular subtype } & 0.066 \\
\hline Luminal A vs. TNBC & $0.80(0.29-2.21)$ & 0.671 \\
\hline Luminal B vs. TNBC & $0.43(0.12-1.51)$ & 0.186 \\
\hline HER2+ vs. TNBC & $4.28(0.86-21.39)$ & 0.077 \\
\hline TILs (high vs. low) & $0.43(0.18-1.05)$ & 0.064 \\
\hline iTILs (high vs. low) & $0.41(0.10-1.76)$ & 0.232 \\
\hline sTILs (high vs. low) & $0.41(0.16-1.04)$ & 0.061 \\
\hline APOBEC3B (high vs. low) & $0.99(0.42-2.32)$ & 0.975 \\
\hline APOBEC3B and TILs (hl vs. hh) & $0.27(0.08-0.88)$ & 0.030 \\
\hline \multicolumn{3}{|l|}{ Multivariate } \\
\hline Grade (1 vs. 2/3) & $0.21(0.08-0.56)$ & 0.002 \\
\hline Tumor size, $\mathrm{cm}(>5$ vs. $\leq 5)$ & $6.25(1.35-29.03)$ & 0.019 \\
\hline Lymph node (+ vs. -) & $4.05(1.14-11.94)$ & 0.011 \\
\hline TNM stage (II/III vs. I) & $0.43(0.07-2.51)$ & 0.346 \\
\hline HER2 (+ vs. -) & $2.09(0.56-7.73)$ & 0.271 \\
\hline APOBEC3B and TILs (hl vs. hh) & $0.65(0.45-0.95)$ & 0.024 \\
\hline
\end{tabular}

DFS, disease-free survival; HR, hazard ration; IDC, invasive ductal carcinoma; ER, estrogen receptor; PR, progesterone receptor; HER2, human epidermal growth factor receptor-2; TILs, tumor infiltrating lymphocytes; iTILs, intratumoral infiltrating lymphocytes; sTILs, tumor infiltrating lymphocytes in stroma; TILs, tumor infiltrating lymphocytes; APOBEC3B, apolipoprotein B mRNA editing enzyme catalytic subunit $3 \mathrm{~B}$; hl, the group with high APOBEC3B protein expression and low levels of TILs; hh, the group with high APOBEC3B protein expression and high levels of TILs.

Fig. 5A and B). High APOBEC3B mRNA expression levels were also indicated to be associated with programmed cell death-ligand 1,PD-L1 (CD274) expression $(\mathrm{P}=0.0001$; Fig. 5C), which is a target for immunotherapy. Overall, these results suggest that $\mathrm{APOBEC} 3 \mathrm{~B}$ expression may be a novel predictor of immunotherapy response.

\section{Discussion}

Breast cancer is the most common malignancy in women as well as the leading cause of cancer-associated mortality in women worldwide (1). In 2018, there were 2,088,849 newly-diagnosed cases of breast cancer and 626,679 cancer-associated mortalities worldwide (1). Despite advancements made in the development of novel treatments, and improved survival of patients with breast cancer, the number of reported cases of recurrence and/or metastasis remains high. Previous studies have highlighted breast cancer as a heterogeneous disease, which is comprised of several somatic mutations, including those in PI3KCA, P53 and ESR1 $(2,3,6,2,8)$. Discovering novel biomarkers for these mutations may prove beneficial in the development of treatments for breast cancer. APOBEC3B has been demonstrated to induce somatic mutations, and thus may be a useful biomarker (15-21). Furthermore, previous studies have reported that APOBEC3B mRNA expression is upregulated in cancer tissues compared with normal tissues, including breast cancer $(12-17,20)$. APOBEC3B mRNA expression has also been associated with poorer RFS and OS rates in ER+ breast cancer (22-25). The present study analyzed APOBEC3B protein expression in patients with breast cancer and investigated its association with the immune system. 
A
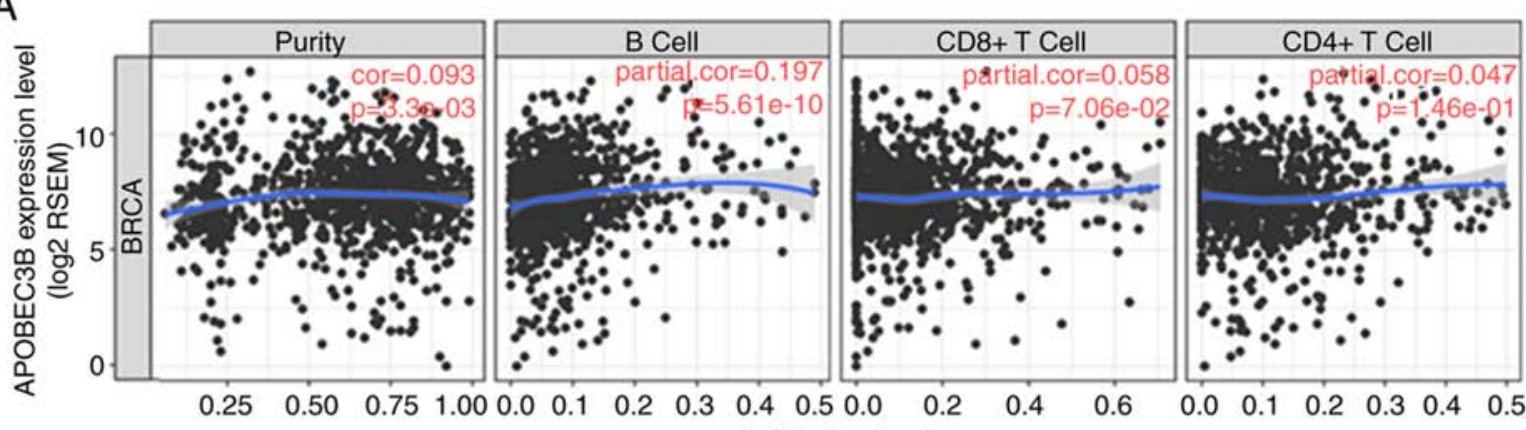

B
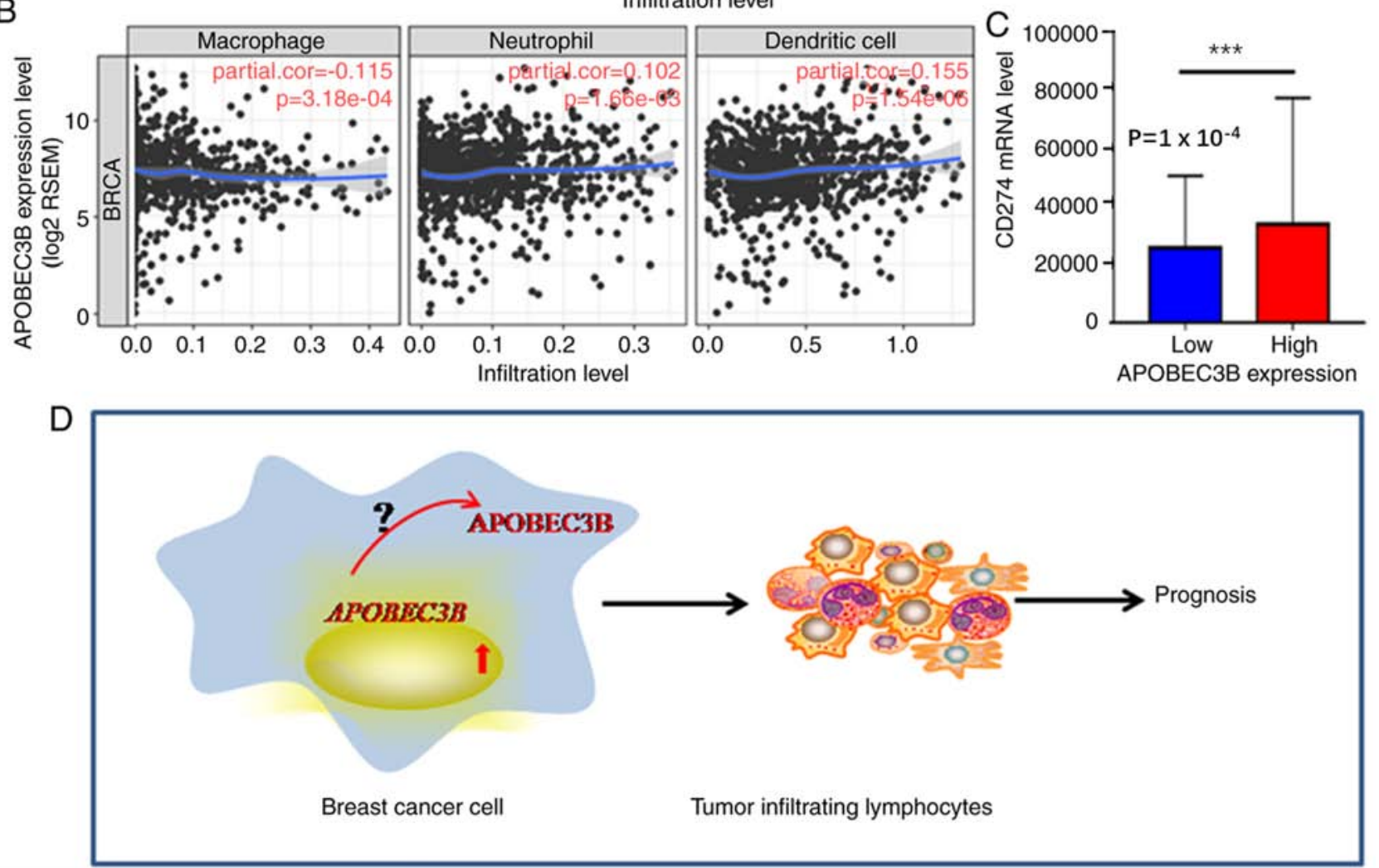

Figure 5. Associations between APOBEC3B mRNA expression and (A) purity, B-cells, CD8+ T-cells, CD4+ T-cells, (B) macrophages, neutrophils, dendritic cells and (C) CD274 mRNA. (D) Schematic representation demonstrating the association between APOBEC3B, tumor infiltrating lymphocytes and prognosis for breast cancer. ${ }^{* * *} \mathrm{P}<0.05$. APOBEC3B, apolipoprotein B mRNA editing enzyme catalytic subunit $3 \mathrm{~B}$.

The results demonstrated that APOBEC3B protein levels were similar to mRNA levels, and were associated with ER and PR status. However, the results did not detect an association between APOBEC3B protein levels and DFS or OS time, which differs from previous studies $(26,32)$. This may be due to the fact that APOBEC $3 \mathrm{~B}$ mRNA and protein levels are not consistent in breast cancer and that APOBEC3B has different roles depending on its surroundings. Rüder et al (26) reported that patients with high-grade serous ovarian carcinoma that are negative for both APOBEC3B mRNA and protein expression, were associated with poor progression-free survival (PFS) time; however, in multivariate analysis, only APOBEC3B mRNA expression was demonstrated to be an independent factor for PFS time. Furthermore, only APOBEC3B cytoplasmic staining indicated a trend towards improved PFS time (26). The difference in results compared with previous studies may be due to the small sample size used in the present study. Future studies are required with larger sample sizes, in order to confirm these observations. Analyses using publicly available data demonstrated that APOBEC3B mRNA expression levels were associated with the prognosis of Luminal breast cancer, particularly the Luminal A subtype. This was the case whether the patient received endocrine therapy or no treatment; however, it was not the case for patients treated using chemotherapy. Taken together, the results suggest that the prognostic value of APOBEC3B mRNA expression levels is valuable in low-risk patients. Consistent with the findings of the present study, previous studies have demonstrated that APOBEC3B mRNA expression levels are associated with the survival of ER+ and lymph node negative patients $(23,24)$. Furthermore, overexpression of APOBEC3B in MCF7 has been reported to induce tamoxifen resistance (25), which may explain the poor prognosis of these patients. A previous study demonstrated that high APOBEC 3 B mRNA expression indicates an improved response rate to neoadjuvant chemotherapy in breast cancer, particularly triple negative 
breast cancer; however, no significant effect was observed on the prognosis (33). This suggests that APOBEC3B mRNA expression levels may serve a predictive role for chemotherapy sensitivity in some breast cancer subtypes, however, future studies are required to determine the exact predictive value of APOBEC3B.

APOBEC3B has been reported to serve vital roles in the immune system $(13,22,26)$, which include TILs. TILs play important roles in the prediction and prognosis in breast cancer. Moreover, immunotherapy maybe effective in breast cancer. However, to the best of our knowledge, no study has analyzed the association between APOBEC3B expression and TILs in breast cancer. The results demonstrated that APOBEC 3B protein expression levels were positively associated with lymphocytes in breast cancer tissue, particularly lymphocytes within the stroma. Such associations have been demonstrated to improve the survival and pathological complete response in breast cancer (34). The precursor study to the present study reported that different types of lymphocyte serve varying roles in the prediction and prognosis of breast cancer $(34,35)$. Despite failure to detect an association between APOBEC3B protein expression levels and lymphocyte subtypes, the present study demonstrated that high APOBEC3B protein expression was associated with increased TIL levels. Furthermore, survival analysis indicated that high APOBEC3B protein expression and high levels of TILs were associated with improved DFS, as an independent factor, compared with the high APOBEC3B protein expression and low levels of TILs. It is plausible that APOBEC3B and TILs may be associated with the active immune status contribute to cancer control, thus making the APOBEC3B protein a notable marker for tumor immune status.

The association between APOBEC3B mRNA expression levels and different subtypes of lymphocyte was also assessed using TCGA and TIMER databases. The results were similar to those of previous studies on ovarian and lung cancer $(13,26)$, demonstrating that high APOBEC3B mRNA and protein expression were associated with TILs in breast cancer, which may contribute to the prognosis of these patients. However, the key factors which are attributed to the translation and location of the APOBEC3B protein, its direct interaction with lymphocytes and the resulting prognosis remain unclear (Fig. 5D), thus further studies are required. Furthermore, APOBEC3B mRNA expression has been reported to be associated with an immune therapeutic response in lung cancer, suggesting its potential as a biomarker (13). Although the predictive value of APOBEC3B, with regards to immune therapy, was not assessed in the present study, the results demonstrated that APOBEC3B mRNA expression was positively associated with PD-L1 expression, which is a known biomarker for immune therapy. This indicates that APOBEC3B may be a promising marker for immunotherapy.

Overall, the results of the present study demonstrated that APOBECB3B mRNA and protein expression levels served varied roles in breast cancer progression. APOBEC3B mRNA expression indicated worse prognosis for patients with breast cancer, particularly patients with the luminal A subtype, while both APOBEC3B protein and mRNA expression were associated with some immune system cells and may have the potential to be developed as a novel prognostic marker for patients with breast cancer.

\section{Acknowledgements}

Not applicable.

\section{Funding}

The present study was supported by the Natural Science Doctoral Funding of Shandong Province (grant nos. ZR2017BH061 and ZR2019BH013), the National Natural Science Foundation of China (grant nos. 81572616 and 81772845), the Qingdao Science and Technology Innovation Plan (grant no. 17-1-1-54-jch), the postdoctoral funding of Qingdao City, youth funding of Qingdao University (Clinical medicine $+\mathrm{X}$ ) and The Affiliated Hospital of Qingdao University.

\section{Availability of data and materials}

The datasets used and/or analyzed during the current study are available from the corresponding author on reasonable request.

\section{Authors' contributions}

YM designed the present study, analyzed the data and drafted the initial manuscript. ML, GN, YoW and JC acquired the clinicopathological data and performed TMA construction. YuW, WC, XL and XW performed the experiments. YZ interpreted the data and HW designed and supervised the present study. All authors have read and approved the final manuscript.

\section{Ethics approval and consent to participate}

All procedures performed in the present study were in accordance with the Ethical Standards of the Institutional and National Research Committee and with The Helsinki Declaration. The present study was approved by the Ethics Committee of the Affiliated Hospital of Qingdao University (approval no. QYFY WZLL 25616) and written informed consent was obtained from all patients prior to the study start.

\section{Patient consent for publication}

Not applicable.

\section{Competing interests}

The authors declare that they have no competing interests.

\section{References}

1. Bray F, Ferlay J, Soerjomataram I, Siegel RL, Torre LA and Jemal A: Global cancer statistics 2018: GLOBOCAN estimates of incidence and mortality worldwide for 36 cancers in 185 countries. CA Cancer J Clin 68: 394-424, 2018.

2. Stephens PJ, Tarpey PS, Davies H, Van Loo P, Greenman C, Wedge DC, Nik-Zainal S, Martin S, Varela I, Bignell GR, et al: The landscape of cancer genes and mutational processes in breast cancer. Nature 486: 400-404, 2012.

3. Cancer Genome Atlas N: Comprehensive molecular portraits of human breast tumours. Nature 490: 61-70, 2012.

4. Hanahan D and Weinberg RA: Hallmarks of cancer: The next generation. Cell 144: 646-674, 2011. 
5. Greenman C, Stephens P, Smith R, Dalgliesh GL, Hunter C, Bignell G, Davies H, Teague J, Butler A, Stevens C, et al: Patterns of somatic mutation in human cancer genomes. Nature 446: $153-158,2007$.

6. Nik-Zainal S, Alexandrov LB, Wedge DC, Van Loo P, Greenman CD, Raine K, Jones D, Hinton J, Marshall J, Stebbings LA, et al: Mutational processes molding the genomes of 21 breast cancers. Cell 149: 979-993, 2012.

7. Alexandrov LB, Nik-Zainal S, Wedge DC, Aparicio SA, Behjati S, Biankin AV, Bignell GR, Bolli N, Borg A, Børresen-Dale AL, et al: Signatures of mutational processes in human cancer. Nature 500: 415-421, 2013.

8. Cancer Genome Atlas Network: Comprehensive molecular portraits of human breast tumours. Nature 490: 61-70, 2012.

9. Smith HC, Bennett RP, Kizilyer A, McDougall WM and Prohaska KM: Functions and regulation of the APOBEC family of proteins. Semin Cell Dev Biol 23: 258-268, 2012.

10. Refsland EW and Harris RS: The APOBEC3 family of retroelement restriction factors. Curr Top Microbiol Immunol 371: 1-27, 2013.

11. Harris RS and Liddament MT: Retroviral restriction by APOBEC proteins. Nat Rev Immunol 4: 868-877, 2004.

12. Ng JCF, Quist J, Grigoriadis A, Malim MH and Fraternali F Pan-cancer transcriptomic analysis dissects immune and proliferative functions of APOBEC 3 cytidine deaminases. Nucleic Acids Res 47: 1178-1194, 2019.

13. Wang S, Jia M, He Z and Liu XS: APOBEC3B and APOBEC mutational signature as potential predictive markers for immunotherapy response in non-small cell lung cancer. Oncogene 37: 3924-3936, 2018.

14. Wen WX, Soo JS, Kwan PY, Hong E, Khang TF, Mariapun S, Lee CS, Hasan SN, Rajadurai P, Yip CH, et al: Germline APOBEC3B deletion is associated with breast cancer risk in an Asian multi-ethnic cohort and with immune cell presentation. Breast Cancer Res 18: 56, 2016.

15. Burns MB, Lackey L, Carpenter MA, Rathore A, Land AM, Leonard B, Refsland EW, Refsland EW, Kotandeniya D, Tretyakova N, et al: APOBEC3B is an enzymatic source of mutation in breast cancer. Nature 494: 366-370, 2013.

16. Middlebrooks CD, Banday AR, Matsuda K, Udquim KI, Onabajo OO, Paquin A, Figueroa JD, Zhu B, Koutros S, Kubo M, et al: Association of germline variants in the APOBEC3 region with cancer risk and enrichment with APOBEC-signature mutations in tumors. Nat Genet 48: 1330-1338, 2016.

17. Roberts SA, Lawrence MS, Klimczak LJ, Grimm SA, Fargo D, Stojanov P, Kiezun A, Kryukov GV, Carter SL, Saksena G, et al: An APOBEC cytidine deaminase mutagenesis pattern is widespread in human cancers. Nat Genet 45: 970-976, 2013.

18. Harris RS: Molecular mechanism and clinical impact of APOBEC3B-catalyzed mutagenesis in breast cancer. Breast Cancer Res 17: 8, 2015.

19. Zhang Y, Delahanty R, Guo X, Zheng W and Long J: Integrative genomic analysis reveals functional diversification of APOBEC gene family in breast cancer. Hum Genomics 9: 34, 2015.

20. Kuong KJ and Loeb LA: APOBEC3B mutagenesis in cancer. Nat Genet 45: 964-965, 2013.

21. Kanu N, Cerone MA, Goh G, Zalmas LP, Bartkova J, Dietzen M, McGranahan N, Rogers R, Law EK, Gromova I, et al: DNA replication stress mediates APOBEC 3 family mutagenesis in breast cancer. Genome Biol 17: 185, 2016.

22. Cescon DW, Haibe-Kains B and Mak TW: APOBEC3B expression in breast cancer reflects cellular proliferation, while a deletion polymorphism is associated with immune activation. Proc Natl Acad Sci USA 112: 2841-2846, 2015.
23. Sieuwerts AM, Willis S, Burns MB, Look MP, Meijer-Van Gelder ME, Schlicker A, Heideman MR, Jacobs H, Wessels L, Leyland-Jones B, et al: Elevated APOBEC3B correlates with poor outcomes for estrogen-receptor positive breast cancers. Horm Cancer 5: 405-413, 2014

24. Tsuboi M, Yamane A, Horiguchi J, Yokobori T, Kawabata-Iwakawa R, Yoshiyama S, Rokudai S, Odawara H, Tokiniwa H, Oyama T, et al: APOBEC3B high expression status is associated with aggressive phenotype in Japanese breast cancers. Breast Cancer 23: 780-788, 2016.

25. Law EK, Sieuwerts AM, LaPara K, Leonard B, Starrett GJ, Molan AM, Temiz NA, Vogel RI, Meijer-van Gelder ME, Sweep FC, et al: The DNA cytosine deaminase APOBEC3B promotes tamoxifen resistance in ER-positive breast cancer. Sci Adv 2: e1601737, 2016.

26. Rüder U, Denkert C, Kunze CA, Jank P, Lindner J, Jöhrens K, Kulbe H, Sehouli J, Dietel M, Braicu E and Darb-Esfahani S: APOBEC3B protein expression and mRNA analyses in patients with high-grade serous ovarian carcinoma. Histol Histopathol 34: 405-417, 2018.

27. Edge SB, Byrd DR, Compton CC, Fritz AG, Greene FL and Trotti A (eds): AJCC cancer staging manual (7th edition). Springer (ed.), New York, pp345-376, 2010.

28. Chang W, Gao X, Han Y, Du Y, Liu Q, Wang L, Tan X, Zhang Q, Liu Y, Zhu Y, et al: Gene expression profling-derived immunohistochemistry signature with high prognostic value in colorectal carcinoma. Gut 63: 1457-1467, 2014

29. Gao XH, Liu QZ, Chang W, Xu XD, Du Y, Han Y, Liu Y, Yu ZQ, Zuo ZG, Xing JJ, et al: Expression of ZNF148 in diferent developing stages of colorectal cancer and its prognostic value: A large Chinese study based on tissue microarray. Cancer 119: 2212-2222, 2013.

30. Salgado R, Denkert C, Demaria S, Sirtaine N, Klauschen F, Pruneri G, Wienert S, Van den Eynden G, Baehner FL, Penault-Llorca F, et al: The evaluation of tumor-infiltrating lymphocytes (TILs) in breast cancer: Recommendations by an International TILs Working Group 2014. Ann Oncol 26: 259-271, 2015.

31. Li B, Severson E, Pignon JC, Zhao H, Li T, Novak J, Jiang P, Shen H, Aster JC, Rodig S, et al: Comprehensive analyses of tumor immunity: Implications for cancer immunotherapy. Genome Biol 17: 174, 2016.

32. Du Y, Tao X, Wu J, Yu H, Yu Y and Zhao H: APOBEC3B up-regulation independently predicts ovarian cancer prognosis: A cohort study. Cancer Cell Int 18: 78, 2018.

33. Fujiki Y, Yamamoto Y, Sueta A, Yamamoto-Ibusuki M, Goto-Yamaguchi L, Tomiguchi M, Takeshita T and Iwase H: APOBEC3B gene expression as a novel predictive factor for pathological complete response to neoadjuvant chemotherapy in breast cancer. Oncotarget 9: 30513-30526, 2018.

34. Mao Y, Qu Q, Chen X, Huang O, Wu J and Shen K: The Prognostic value of tumor-infiltrating lymphocytes in breast cancer: A Systematic review and meta-analysis. PLoS One 11: $\mathrm{e} 0152500,2016$.

35. Mao Y, Qu Q, Zhang Y, Liu J, Chen X and Shen K: The value of tumor infiltrating lymphocytes for predicting response to neoadjuvant chemotherapy in breast cancer: A systemic review and meta-analysis. PLoS One 9: e0115103, 2014.

This work is licensed under a Creative Commons Attribution-NonCommercial-NoDerivatives 4.0 International (CC BY-NC-ND 4.0) License. 